\title{
La Brucellose Bovine au Tchad
}

\author{
par P. PERREAU
}

Dans un travail publié dans la Revue d'Elevage et de Médecine Vétérinaire des Pays Tropicaux (20). Sacquet a signalé l'existence et l'importance de la brucellose bovine au Tchad, en particulier sur les animaux de la région de Fort-Lamy.

En 1955, nous avons étendu cette enquête à diverses régions $\mathrm{du}$ Territoire. 2.911 examens individuels ont été pratiqués, dont 1.933 séroagglutinations et 978 ring-tests. Les résultats obtenus ont permis de préciser l'importance de la maladie dans les zones prospectées et ils serviront à établir la carte d'une affection qui semble susceptible de constituer, pour le cheptel du Tchad.aussi bien que pour la population humaine, grande consommatrice de lait cru, une maladie d'avenir suivant l'expression de Charles Nicolle.

Trois régions ont été prospectées : Chari-Baguirmi, Ouaddaï et Mayo-Kebbi; le bétail d'environ 40 villages disséminés dans ces régions a fait l'objet d'un exannen systénalique. Il était d'ailleurs intéressant de pouvoir comparer. l'importance épidémiologique de la brucellose bovine dans les troupeaux de races différentes, appartenant à des groupes ethniques bien individualisés ayant chacun leur mode d'élevage propre, tels que les Arabes du Ouaddaĩ et du Chari-Baguirmi, les Bororos, les Foulbés et les sédentaires du sud, Toubouris et Bananas.

Le Inarché aux bestiaux de Forl-Lamy nous a fourni en outre un nombre important d'animaux de toutes origines.

Seuls des tests individuels ont été pratiqués, afin de préciser le pourcentage de sujets atteints au sein de chaque troupeau; la préférence a été donnée à la séro-agglutination et le ring-test n'a été utilisé que dans les circonstances où les sérums ne pouvaient être rapidement envoyés au laboratoire.

Quelques renseignements d'ordre clinique sont venus s'ajouter aux rares observations déjà faites.

Les résultats généraux de cette enquête peuvent être rassemblés dans le tableau ci-contre :

Bien que ces chiffres n'aient qu'une valeur relative parce qu'ils ne constituent que des résultats partiels, ils suffisent à donner un ordre de grandeur moyen de l'infection brucellique.

Le taux moyen d'animaux infectés est de $12 \%$; mais des différences sensibles apparaissent selon les régions et les troupeaux.
Le nord de la région de Fort-Lamy et la bordure est du lac Tchad (district de Massakory et de Moussoro) apparaissent fortement contaminés.

Le taux d'animaux atteints est souvent très inégal entre villages d'une même région; bien que le district de Léré ne foumisse qu'un chiffre moyen de $12,2 \%$, dans un village de cette région nous

\begin{tabular}{|c|c|c|}
\hline RÉGIONS & DISTRICTS & $\%$ d'animaux \\
\hline $\begin{array}{l}\text { Chari-Baguirmi } \\
\text { (moyenne }: 12 \% \text { ) }\end{array}$ & $\begin{array}{c}\text { Fort-Iamy } \\
\text { Massakory } \\
\text { Massénya }\end{array}$ & $\begin{array}{l}14 \\
23,8 \\
10,9\end{array}$ \\
\hline $\begin{array}{c}\text { Ouaddaï } \\
\text { (moyenne : } 9,3 \% \text { ) }\end{array}$ & Abécher & 8,3 \\
\hline $\begin{array}{c}\text { Kanem } \\
\text { (moyenne : 16, } 9 \% \text { ) }\end{array}$ & Moussoro & 16.9 \\
\hline $\begin{array}{c}\text { Mayo-Kebbi } \\
\text { (moyenne : } 10,7 \% \text { ) }\end{array}$ & $\begin{array}{c}\text { Bongor } \\
\text { Léré } \\
\text { Fianga }\end{array}$ & $\begin{array}{r}8,4 \\
12,2 \\
7,4\end{array}$ \\
\hline
\end{tabular}

avons trouvé $41 \%$ de tests individuels positifs, et seulement $4 \%$ dans un second village proche de quelques kilomètres : dans les conditions actuelles de l'élevage au Tchad, et en matière' de brucellose bovine, le foyer épidémiologique n'est ni l'animal isolé, ni le troupeau plus ou moins grand d'un seul propriétaire, mais bien le troupeau entier du village ou du groupement d'éleveurs : le férik ou le cachimbet, lorsqu'il s'agit de transhumants, grands ou petits, le qualificatif précisant l'étendue du déplacement, lequel peut atteindre jusqu'à $800 \mathrm{~km} \mathrm{~N}$.-S.

En effet, les animaux y sont en contact, fréquemment rassemblés, quelquefois sous un gardiennage commun, buvant à la même mare et bien souvent parqués pour la nuit tous ensemble sur la place centrale du village ou du férik.

La brucellose bovine semble donc sévir sur l'ensemble du Territoire et atteindre de façon identique zébus arabes, Borroros, Foulbés, race Kouri et bétail du Logone. 


$$
* *
$$

L'avortement, signe clinique majeur de la brucellose, est-il observé?

On peut affirmer que, dans deux régions au moins, les avortements sont constatés, associés ou non à d'autres signes: hygromas et synovites.

Chez les éleveurs arabes de la région d'Abécher, agglutinations positives, constatent quelques avortements, et chaque propriétaire connait fort bien dans son troupeau la vache qui avorte parfois deux fois de suite. Ils ne peuvent établir naturellement un rapport entre ces accidents et une maladie infectieuse; mais les hygromas et les synovites, relativement fréquents dans ces mêmes troupeaux, quelquefois associés à un avortement sur le même

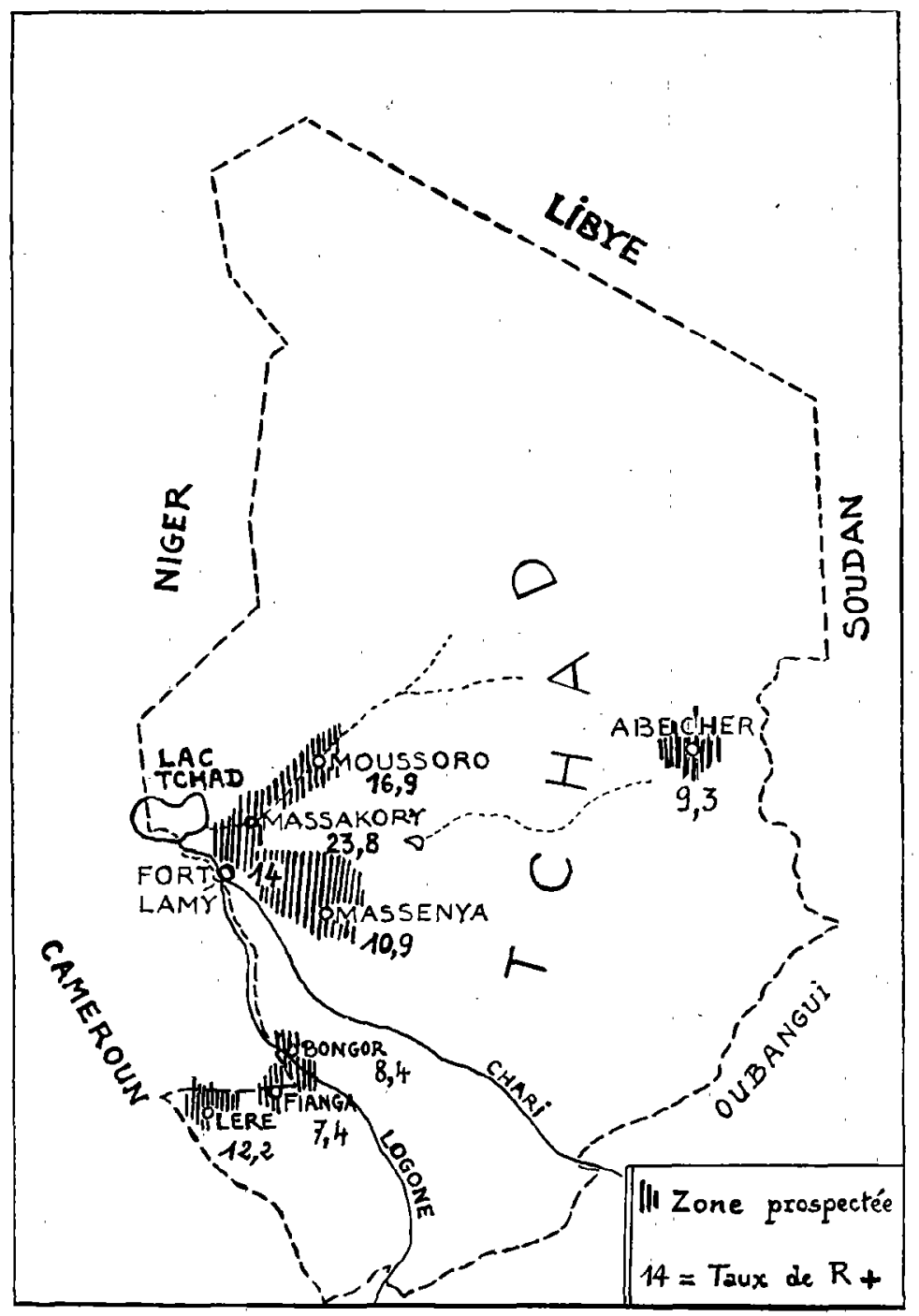

plusieurs de ces accidents nous ont été signalés, nous ne pouvons bien entendu les attribuer exclusivement à la brucellose, mais nous avons pu retrouver une des vaches, dont l'avortement remontait à quelques semaines, et son sérum agglutinait Brucella abortus bovis au $1 / 5000$.

Les éleveurs Foulbés des environs de Binder, dont les troupeaux ont fourni un taux élevé de séro- animal, constituent à leurs yeux une entité pathologique à laquelle ils ont donné le nom de «bakélé », gênante surtout parce qu'elle déparo l'animal ou le fait boiter $\left(^{*}\right)$.

Le syndrome est identique à celui décrit en 1948

(*) La maladie a été décelée dès 1908 par Pécaud (N.D.L.R.). 
par Camara (5) en A.O.F.; les animaux atteints de "bakélé » ont souvent, mais pas toujours, une séro-agglutination positive.

Il est hors de doute qu'un certain nombre d'avortements passent inaperçus, même chez ces Foulbés attentifs à leurs troupeaux, mais il n'en reste pas moins vrai que ces accidents semblent bien rares par rapport au nombre assez considérable d'animaux infectés.

Sans doute est-il nécessaire, ici aussi, de faire une distinction entre la brucellose-infection, révélèe seulement par le séro-diagnostic, et la brucellosemaladie avec son syndrome complet.

Les longs délais s'écoulant souvent entre deux gestations chez les animaux vivant en milieu tropical permettent peut-être à l'organismo de se constituer une immunité suffisante, evitant au moins la répétition d'un premier avortement.

$$
x^{*} *
$$

Il est impossible, dans les conditions actuelles de l'élevage au Tchad, d'apprécier l'incidence économique de la brucellose bovine, car nous possédons trop peu d'éléments pour apprécier des pertes dont l'évaluation dojt être faite en faisant intervenir des considerants fort différents de ceux retenus en pays évolués, à niveau économique élevé.

Par ailleurs, si en raison même des conditions primitives dans lesquelles sont entretenus les animaux, il serait illusoirc do vouloir, à l'heurc actuclle, mettre sur pied un plan de lutte contre cette affection (à l'exception des rares troupeaux privés), il n'en demeure pas moins que c'est là un problème qui ne saurait être perdu de vue, car son importance croîtra au fur et à mesure même de l'évolution du troupeau vers un mode d'élevage amélioré.

Service de Microbiologie, Laboratoire Fédéral de l'Élevage de l'Afrique Équatoriale Française (Directeur : L. Thomé).

\section{BIBLIOGRAPHIE}

(1) BLANCHARD (A.) et SINALY COULIBALY. Recherches sur la brucellose bovine en Haute-Volta (Afrique occidentale française). Rev. Élev. Méd. Pays Trop., 1954, 7, n०3, 153.

(2) BOURGUIGNON (G.), - Le premier cas de fièvre ondulante diagnostiqué bactériologiquement an Congo belge et ses affinités sérologiques avec Brucella abortus. Ann. Soc. Belge Méd. Trop., 1933, 13, 249.
(3) BOURRET (G.). - La fièvre méditerranéenne en A.O.F. - Bull. Soc. Path. Exot., 1910, 3, 490.

(4) CECCALDI (J.) et GUILHAUMOU (F.). - La bra cellose hamaine en A.E.F. Isolement d'une souche de Brucella melitensis à l'occasion du premiex cas constaté an Tchad dans l'Ennedi. Revue des sciences médicales pharmaceutiques et vétérinaires de l'Afrique française libre, $1942,1,11$.

(5) CAMARA (A.). - Le Bakkalé est-il de la brucellose? Bull. Ser. Elev. A.O.F. 1948, 1, 24.

(6) CLEARKIN (P.-A.). - A Review of Recent Litterature of Undulant Fever of Man and Contagious Abortion in Animals. - Kenya Medical Journal, 1921, 1, 333.

(7) ELMES (B.-G.-T.), - Undulant fever in Nigeria. Annals of Tropical Medicine and Parasitology, $1941,35,1$.

(8) FLOCH (H.). - Diagnostic rétrospectif d'un cas de brucellose contractée en Guyane française. - Bull. Soc. Path. Exot., 1939, 32, 824.

(9) FOREST HUDDLESON. - Brucellosis in Man and Animals (1943), New-York. The Commonwealth Fund - E.L. Hildreth and Co. Inc., page 184.

(10) KAPLAN (M.-M.). - Les connaissances actuelles sur la brucellose. Bull. O.M.S., 1951, 3, 309-322.

(11) KAPLAN (Mi.-M.). — La brucellose : exposé succinct sur l'état actuel de la question. Groupe OMSFAO d'experts de la brucellose, 1950.

(12) LEBLANC (J.), LAMBILLON (J.) et DENISOFF (N.). - Note préliminaire au sujet de 4 cas de bracellose identifiée au Centre médical de la Formulac, au Kivu (Congo belge). Ann. Soc. belge Med. Trop., 1939, 19, 197.

(13) MALBRANT (R.), CECCALDI (H.), GUILHAUMOU (J.) et GROSPERRIN (R.). — Brucellose bovine, trypanosomiase et prémunition. Revue des Sciences médicales, pharmaceutiques et vétérinaires de l'Afrique française libre, 1943, 2, 199-206.

(14) MERCIFR (T.) et BORDES (L.-A.). - Deux cas de mélitococcie contractée en Indochine et en Afrique occidentale française. Bull. Soc. Patho. exo., 1953, 46, 211.

(15) MERLE (F.). - Apparition de la fièvre de Malte au Niger. Bull. Soc. Patho. exo., 1953, 46, 211. 
(16) MOUSTARDIER, - Premier cas de mélitoccocie observé en A.E.F. Revue Sciences médicales, pharmaceutiques et vétérinaires de l'Afrique française libre, $1942, \mathbf{1}, 3$.

(17) PELTIER (M.), ARQUIE (E.), FABRE (P.), DURIEUX (C.) et JONCHĖRES. - Brucellose humaine en Afrique occidentale française. Isolement d'une souche de Brucella melitensis. Bull. Soc. Path. Exo., 1938, 31, 575.

(18) PERCHER (G.) et NOEL (G.). . Une nouvelle " espèce " de Brucella : Brucella intermedia. Ann. Inst. Pasteur, 1952, 83, 814-815.

(19) RENOUX (G.). - Note sur la fièvre ondulante au Ruanda-Urandi. Ann. Soc. belge Méd. Trop., 1936, 16, 217.

(20) SACQUET (E.), - La brucellose bovine au Tchad (note préliminaire). Rev. Fil. Méd. Vét. Pays Trop., (1955), 8, 5-7.

(21) SICLE (A.), ROBIN (Ch.) et PERNARD (Y.). - $\boldsymbol{A}$ propos de 2 cas de mélitococcie contractés au Soudan français et provoqués par Brucella melitensis. Bull. Soc, Patho. Exo., 1939, 32, 409.

(22) WRIGHT (F.-J.), COOKE (E.-R.-N.) et D'SOUZA (J.) St. A.-M. - Observations on Brucellosis in Kenya. Trans. Roy. Soc. Trop. Med. Hyg., $1953,47,117-129$.

\section{Rapports des Services vétérinaires}

Congo belge. - Gouvernement général. Agriculture et Colonisation. Rapport vétérinaire pour 1938, p. 10; pour 1944, p. 43.

Colony and Protectorate of Kenya. - Veterinary Department Annual Report., 1950, p. 15.

Mozambique. - Annais dos Serviços de veterinaria e Industria animal, 1947, $n^{\circ} 1$ in Bulletin of Epizootic Diseases of Africa, 1953, 1, p. 202.

Nigeria. - Annual Report of the Veterinary Department for the Year 1949-1950, p. 53; 1948-1949, p. 61 et 62 .

Government of Nothern Rhodesia. - Veterinary Department. Innual Report for the Year 1945, p. 4.

Government of Southern Rhodesia. - Veterinary Department. Annual Report for the Year 1950 in Bull. of Epizootic Diseases of Africa, 1953, 1, p. 183.

Sudan. - Report of the Sudan Veterinary Service for the Years 1947, 1948, 1949, 1950.

Uganda Protectorate. - Annual Report of the Veterinary Department for the Year Ended 31 December 1951 in Bulletin of Epizootic Diseases of Africa, 1953, 1, p. 363.

\section{SUMMARY}

\section{Bovine brucellosis in Tchad}

A survey has shown the prevalence of bovine brucellosis in some areas of Tchad Territory, French Equatorial Africa. The average incidence of infected animals is $12 \%$, the highest incidence being in cattle originating from the Northern area of Fort Lamy and the Eastern shores of Lake Tchad. The importance of the disease is increasing.

\section{RESUMEN}

Brucelosis bovina en Tchad

Este estudio hace destacar la importancia de la brucelosis bovina en las regiones examinadas del Tchad : Chari-Baguirmi, Ouaddaï y Mayo-Kebbi.

El porcentaje de animales infectados es de un $12 \%$. Los rebaños de la región Norte de Fort-Lamy y del borde Este del lago Tchad son los que aparecen más contaminados.

La brucelosis es una enfermedad del porvenir. 